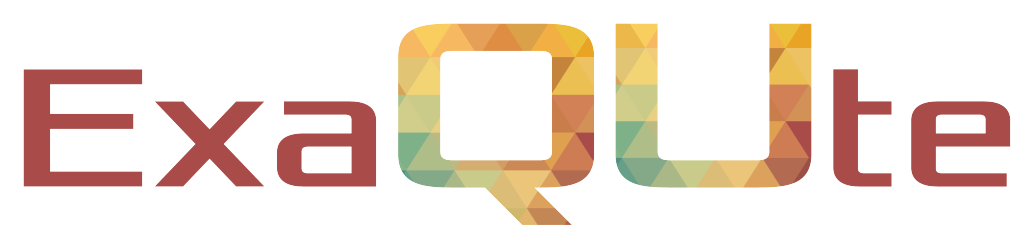

Exascale Quantification of Uncertainties for Technology and Science Simulation

\title{
D6.1 Deterministic optimization software
}

\section{Document information table}

\begin{tabular}{|l|l|}
\hline Contract number: & 800898 \\
\hline Project acronym: & ExaQUte \\
\hline Project Coordinator: & CIMNE \\
\hline Document Responsible Partner: & TUM \\
\hline Deliverable Type: & Report, Other \\
\hline Dissemination Level: & Public \\
\hline Related WP \& Task: & WP6, Task 6.1 \\
\hline Status: & Final version \\
\hline
\end{tabular}




\section{Authoring}

\begin{tabular}{|l|l|l|l|l|}
\hline Prepared by: & Partner & Modified Page/Sections & Version & Comments \\
\hline Authors & TUM & & & \\
\hline Andreas Apostolatos & TUM & & & \\
\hline Brendan Keith & TUM & & & \\
\hline Contributors & & & & \\
\hline & & & & \\
\hline & & & & \\
\hline
\end{tabular}

\section{Change Log}

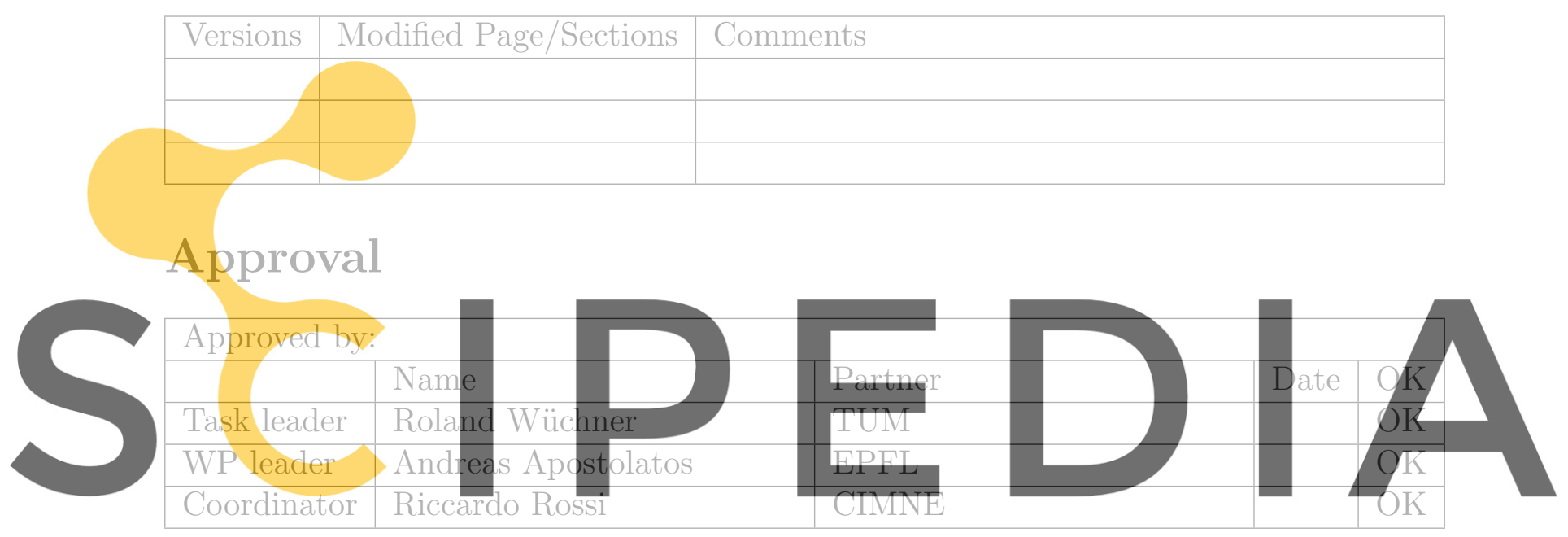

Register for free at https//www.scipedia.com to download the version without the watermark 


\section{Table of contents}

1 Introduction

2 Sensitivity analysis

3 Optimization

4 Example

A API definition and usage
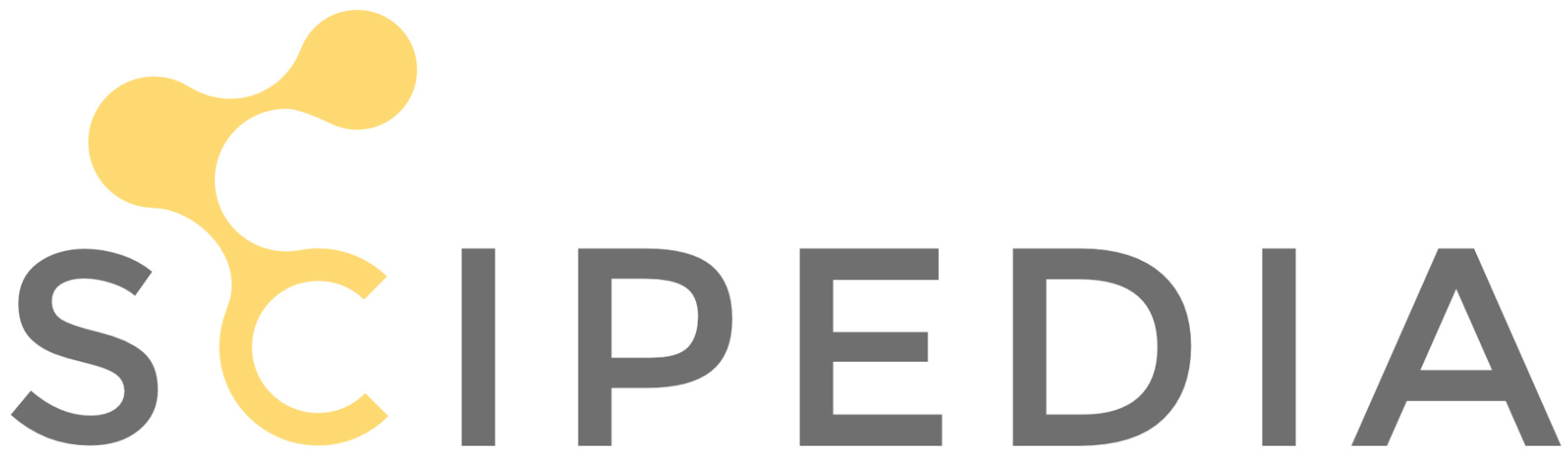

Register for free at https//www.scipedia.com to download the version without the watermark 


\section{List of Figures}

1 Mesh motion computed in KRATOS. . . . . . . . . . . . . . 7

2 Implementational concept of the deterministic algorithms in KRATOS. . . 9

3 Problem placement of a hook for the shape optimization algorithm. . . . . 9

4 Shape optimized hook for mass under the constraint of keeping the strainenergy (stiffness) constant. . . . . . . . . . . . . . . . 10

5 Objective and constraint history through the optimization iterations. . . . 10

$6 \quad$ Sensitivity map at the tenth optimization iteration. . . . . . . . . . . . 11

$7 \quad$ Example of the corresponding calls in the python level for the deterministic optimization algorithms using in KRATOS and an internal analyser. (taken from file./src/run_test.py) . . . . . . . . . . . . . . . . . . . 12

8 Example of the corresponding calls in the python level for the deterministic optimization algorithms using in KRATOS using an external analyser. (taken and slightly modified from file./src/run_test.py) . . . . . . . . . 12

9 Class associated with the internal analyser in KRATOS. (taken from file ./sre/analyzer factory.py) . . . . . . . . . . . . . . . . 13

10 Class associated with the external analyser in KRATOS. (taken from file -/src/analyzer_factory.py)
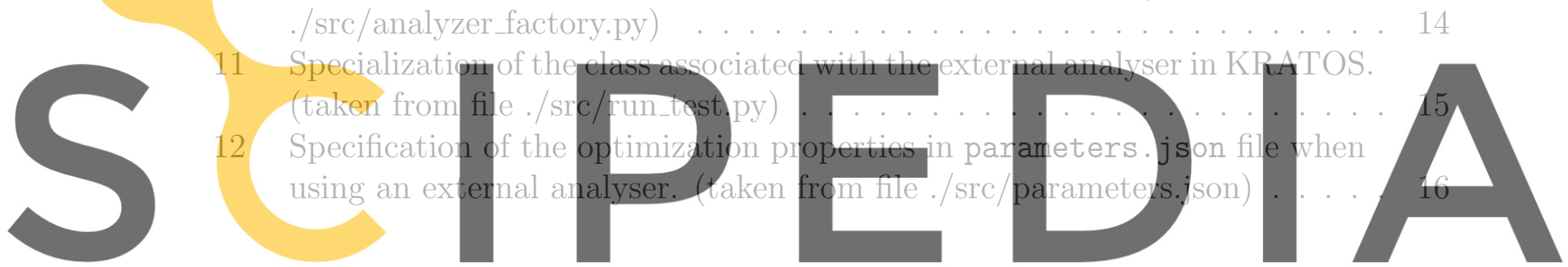

Register for free at https//www.scipedia.com to download the version without the watermark 
Nomenclature / Acronym list

\begin{tabular}{|l|l|}
\hline Acronym & Meaning \\
\hline \hline API & Application Programming Interface \\
\hline ExaQUte & $\begin{array}{l}\text { EXAscale Quantification of Uncertainties for Technology } \\
\text { and Science Simulation }\end{array}$ \\
\hline QoI & Quantity of Interest \\
\hline MC & Monte Carlo \\
\hline MLMC & Multilevel Monte Carlo method \\
\hline C-MLMC & Continuation Multilevel Monte Carlo method \\
\hline HPC & High performance computing \\
\hline PDE & Partial differential equation \\
\hline
\end{tabular}
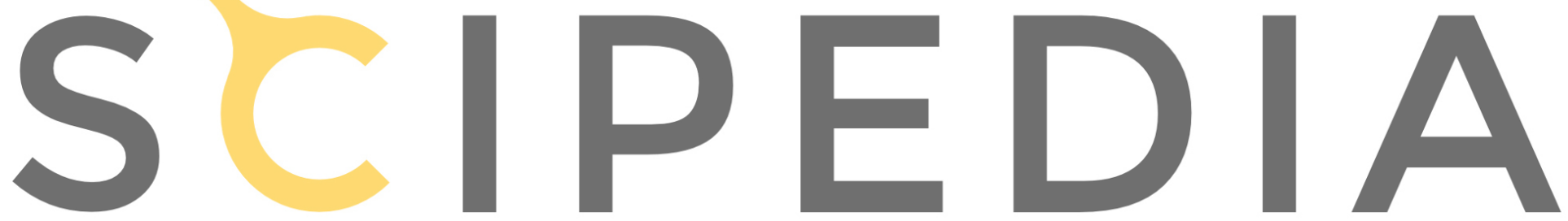

Register for free at https//www.scipedia.com to download the version without the watermark 


\section{Introduction}

This deliverable focuses on the implementation of deterministic optimization algorithms and problem solvers within KRATOS open-source software. One of the main challenges of optimization algorithms in Finite-Element based optimization is how to get the gradient of response functions which are used as objective and constraints when this is not available in an explicit form. The idea is to use local sensitivity analysis to get the gradient of the response function(s)

\section{Sensitivity analysis}

Sensitivity analysis $[4,7,8]$, plays an important role in optimization: It can be used to study the effects of the variation of the input parameters onto the output parameters of the underlying problem. Herein, suitable sensitivity analysis methods for Finite-Element based models are employed: Those include direct or adjoint approaches for sensitivity analysis when the response function depends on the design variable(s) as well as on the state variable(s) which is the case for most responses in structural mechanics such as the nodal displacements, the stress field and the strain-energy relationship. Other types of responses may depend
load factor are treated
functions than design
uations depends on the
approach it is beneficia.
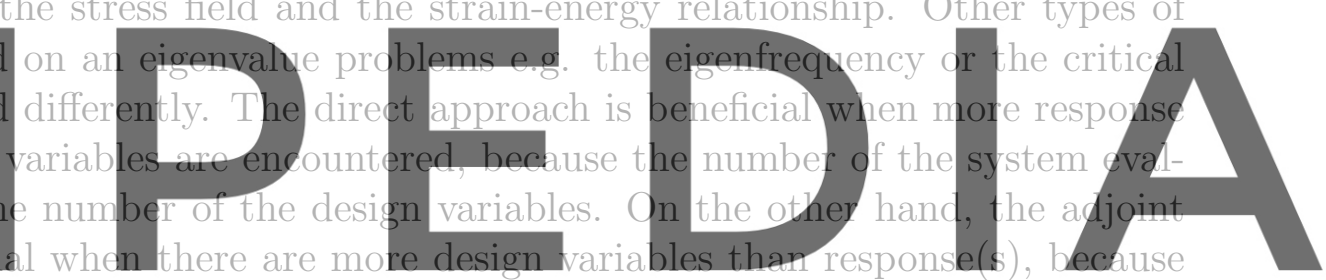

the number of system evaluations depends on the number of responses.

Register for free at https//www.scipedia.com to download the version without the watermark

Finite Element-based optimization involves often more design variables than objectives/constraints e.g. in shape optimization. In KRATOS open-source software the discrete semi-analytic adjoint sensitivity analysis is employed.

Regarding the discrete approach, the response function is firstly discretized and then the derivatives with respect to the discrete parameters are evaluated.

On the contrary, the semi-analytic approach, the analytic procedure is performed up to a certain extent, which is typically confined in the element level, whereas the remaining parts of the sensitivity equation are approximated by a finite difference scheme. The advantages of the semi-analytic versus the analytic approach are,

- Implementation of analytic derivatives of element based data can be omitted, which is in general a very challenging task

- A generic code structure for different kinds of parameters is possible

- It can be used for any arbitrary element formulation and

- It is faster than the analytic approach

whereas the disadvantages in principle are, 
- Reduced accuracy

- Challenging of finding an "optimal" disturbance measure.

The herein employed workflow for the adjoint sensitivity analysis in KRATOS includes,

- Solving the primal problem to determine the state variables

- Solving the adjoint problem for each response of the primal problem which presents the main effort in adjoint sensitivity analysis and which involves solving a linear system even for geometric nonlinear problems. The adjoint problem is independent of the traced design variables and it solely depends on the response. The solution of this problem are the so-called adjoint variables.

- Solving for the sensitivities in a post-processing step.

\section{Optimization}

The Vertex Morphing method is employed within the optimization solution procedure in

KRATOS for the sensitivity filtering, see in $[3,6]$. This method is especially important as

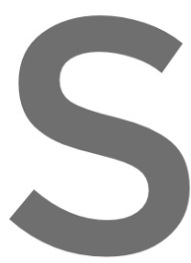

in shape optimizatio

of this kind of proble

Finite Element-based
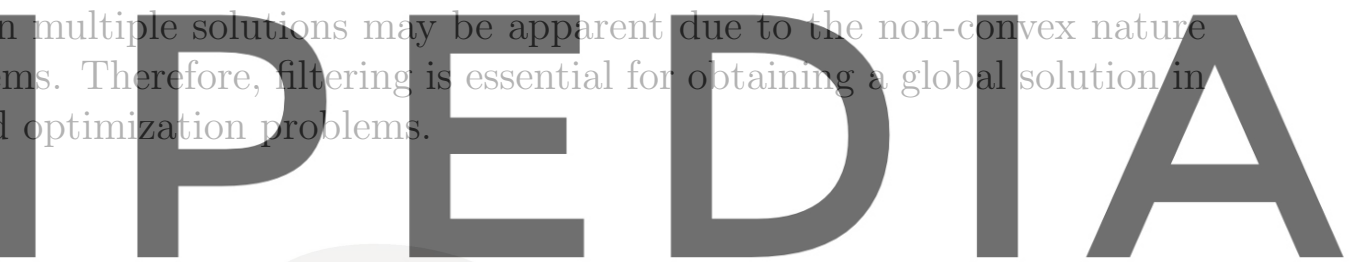

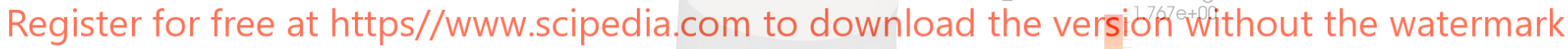

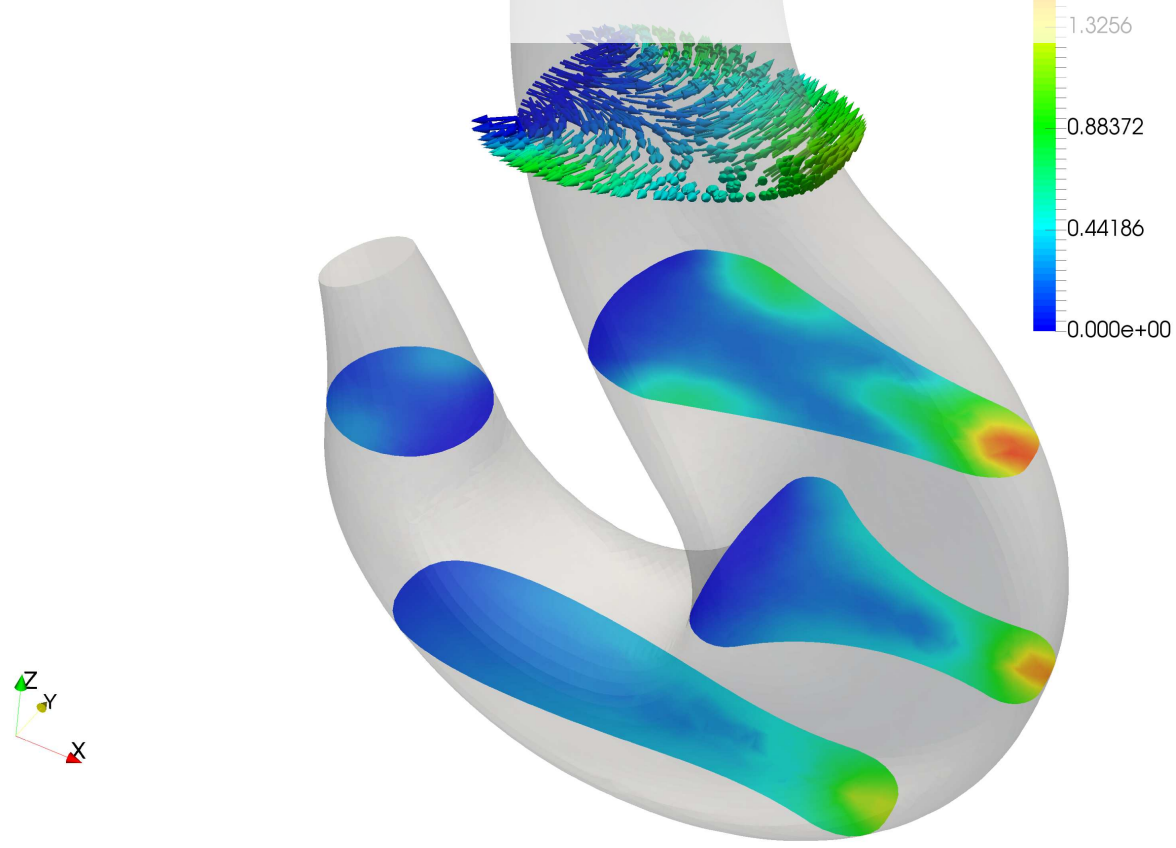

Figure 1: Mesh motion computed in KRATOS. 
The implementation in KRATOS involves both unconstrained and constrained problems. Especially for the constrained problems, one constraint is at the moment available whereas up to ten constraints can be used in principle. Concerning the unconstrained method, a simple steepest decent approach with basic line search is included. Regarding the optimization with one constraint, a projection method is herein implemented [5]. In what concerns optimization with several constraints, an experimental trust region method is being implemented. The employed optimization algorithms can be applied to solid and shell structures.

The current implementation in KRATOS can handle millions of design variables. Different filter functions are included to control the surface continuity. Different filter strategies, both matrix-based and matrix-free, are included depending on the available computer resources. Details on how the filtering affects the design are described in [2]

Several objective functions are implemented, such as the strain-energy, eigenfrequency, mass, stresses etc. Sensitivity analysis is incorporated for each objective function. Regarding objectives which depend on design and state variables, gradient computation through semi-analytic adjoint sensitivity analysis is employed. Moreover, steady-state analysis is herein assumed.
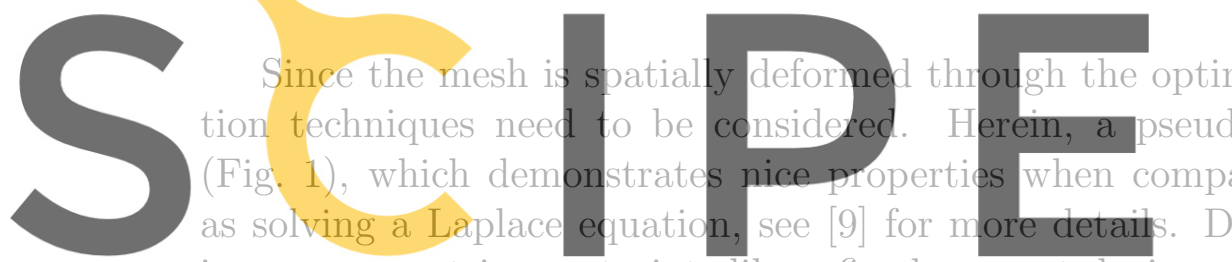

impose geometric constraints like a fixed support during optimization.

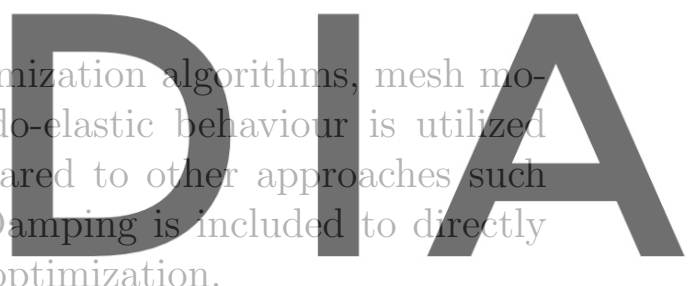

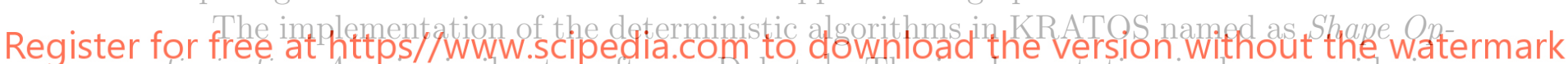
timization App is similar to software Dakota. The implementation is done considering its application to relatively large cases and quite long computational times, which necessitates efficiency and robustness. The latter means that connections between different analyses may be considered (e.g. different adjoint analysis referencing to the results of a single primal analysis or two function values coming from one physical solution, like the lift or drag coefficients in aerodynamics). The latter concept called as analyser-optimisercommunicator (Fig. 2) allows for connections to external solvers, such as demonstrated in $[1]$.

\section{Example}

This section demonstrates the aforementioned algorithms based on a solid structure example. The objective is the minimization of mass of a hook under the constraint of keeping the initial strain-energy (stiffness) of the structure constant (Fig 3). The employed algorithm is herein penalized projection. Figs. in 5 show significant improvement while accurately satisfying the constraint. It is worth noting that the geometry at the converged optimized shape is a very intuitive design. The latter can be explained by the fact that in order to reduce the mass while maintaining the stiffness, one should "extract" material from the bending axis which is exactly what the converged shape exhibits.

\footnotetext{
${ }^{1}$ https://dakota.sandia.gov/
} 


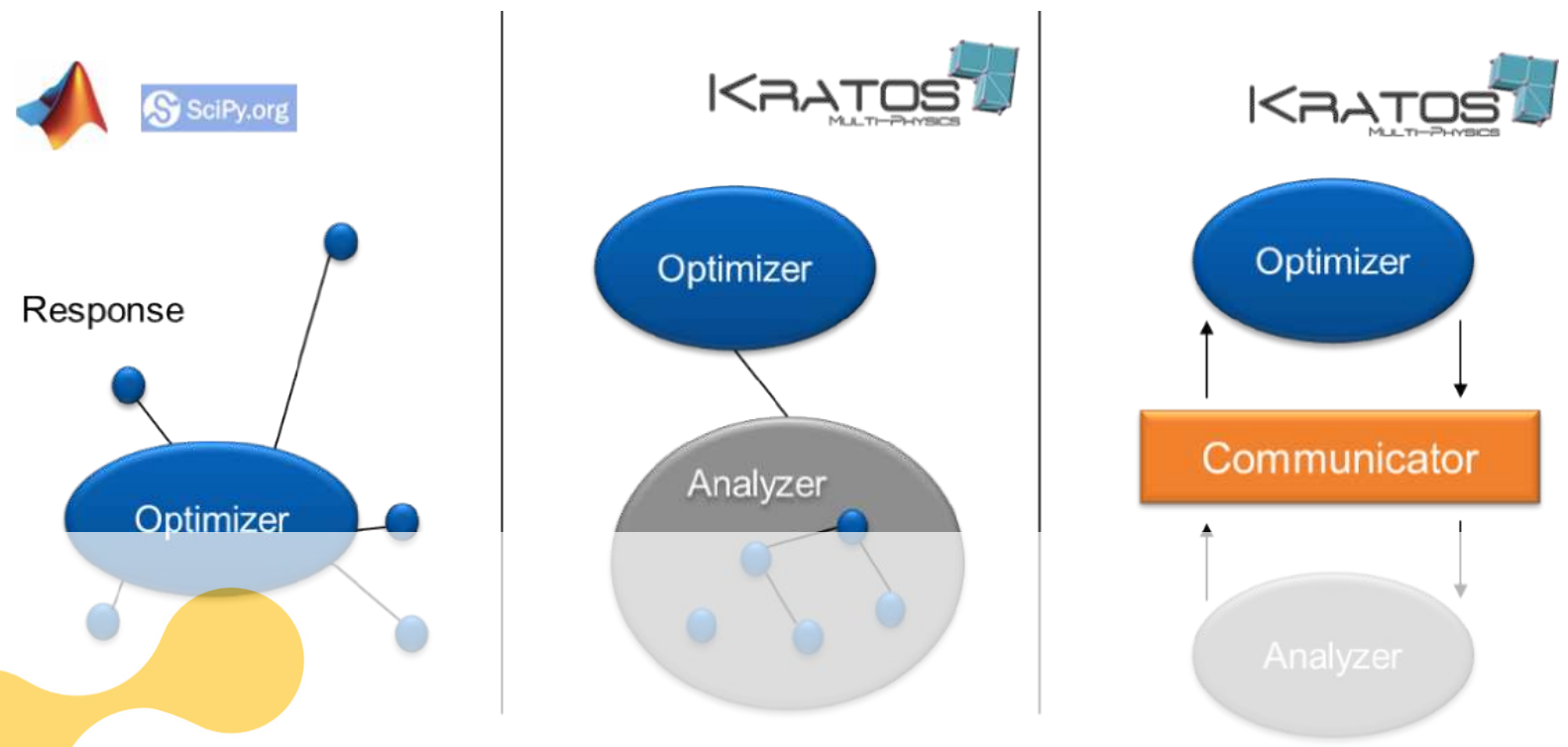

Figure 2: Implementational concept of the deterministic algorithms in KRATOS.
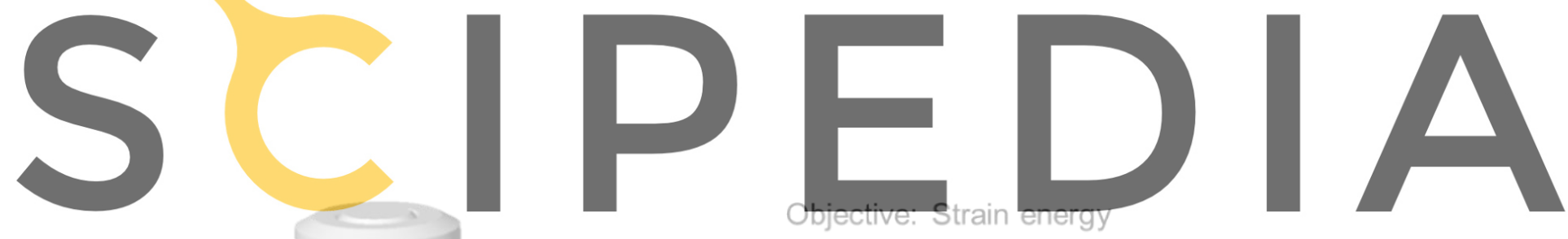

Register for free at https//www.scipedia.com to download the version without the watermark

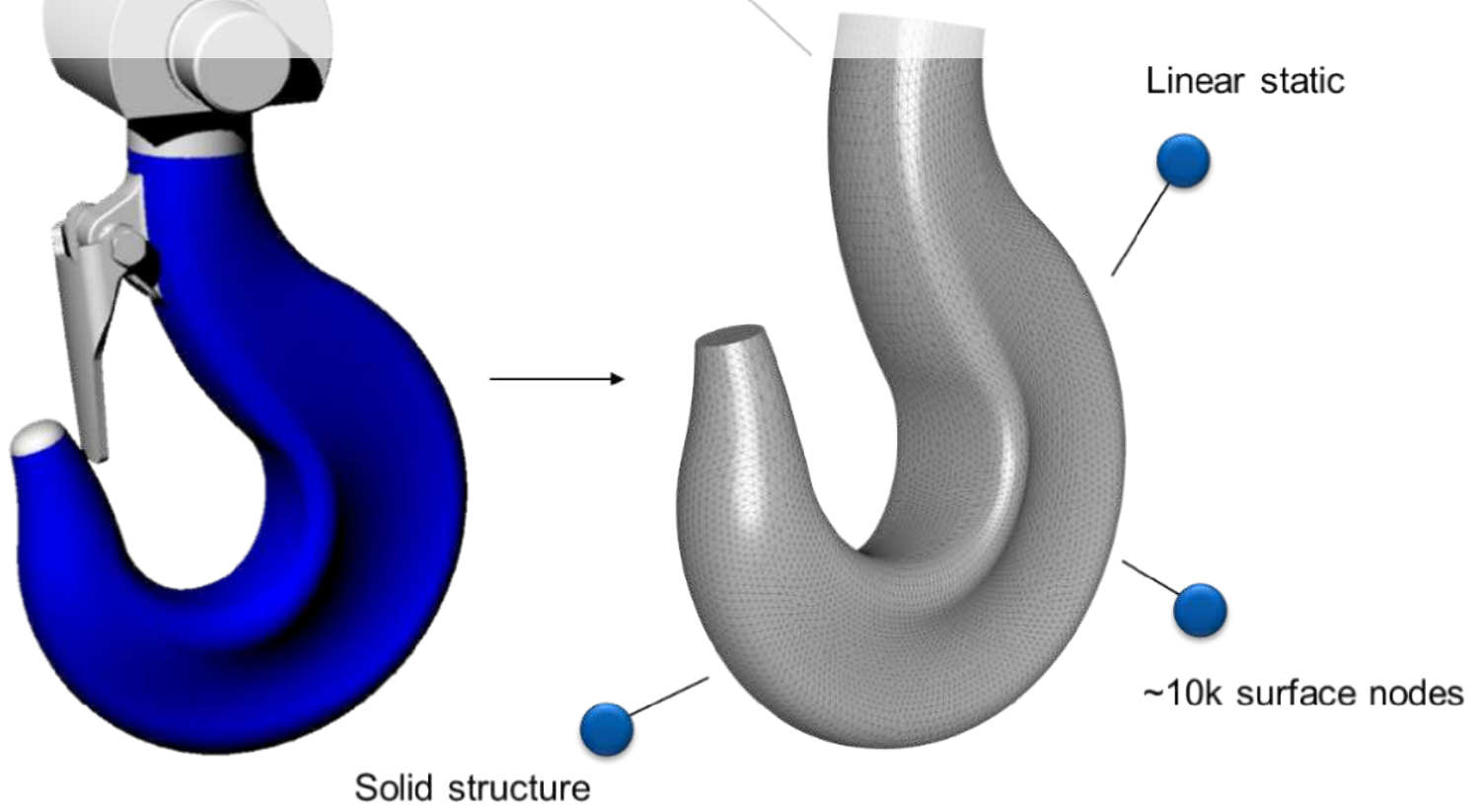

Figure 3: Problem placement of a hook for the shape optimization algorithm. 


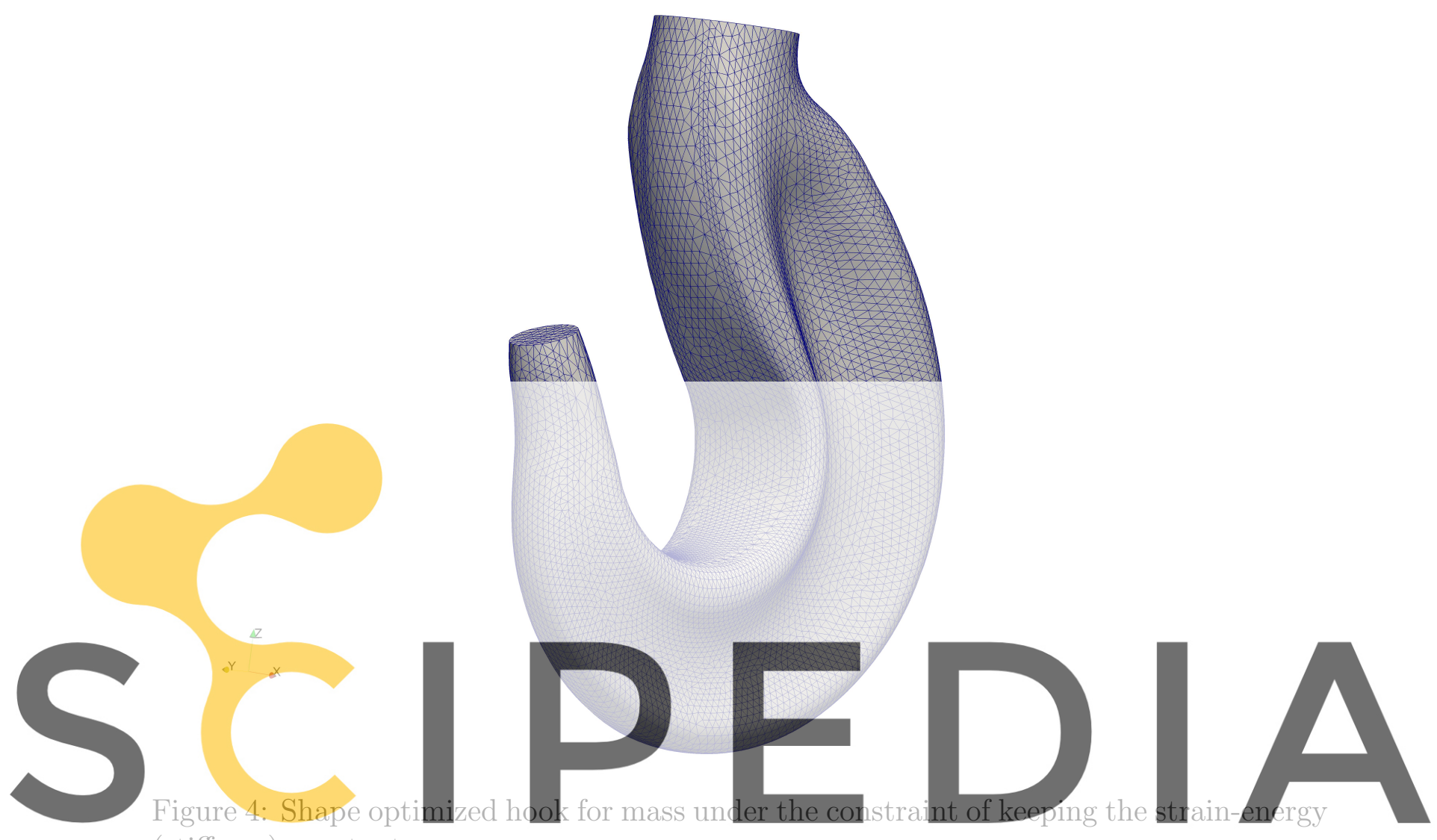

(stiffness) constant.

Register for free at https//www.scipedia.com to download the version without the watermark
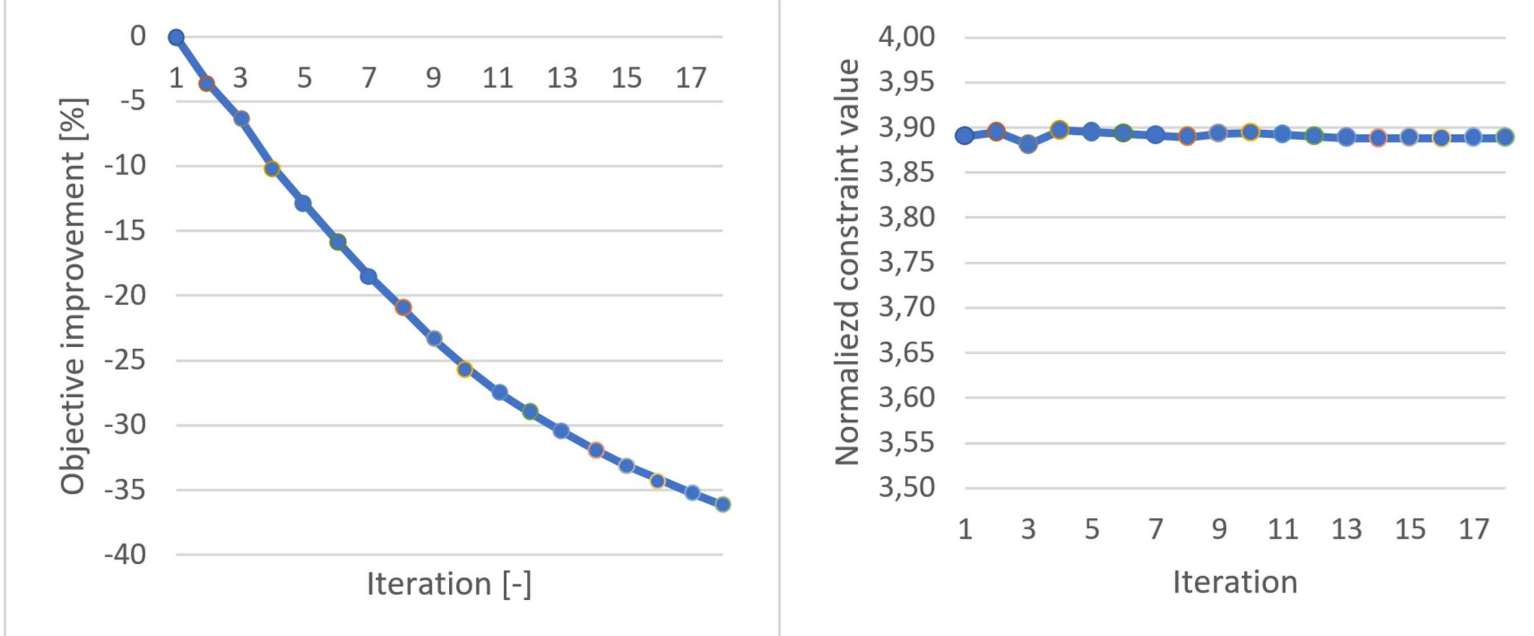

Figure 5: Objective and constraint history through the optimization iterations. 


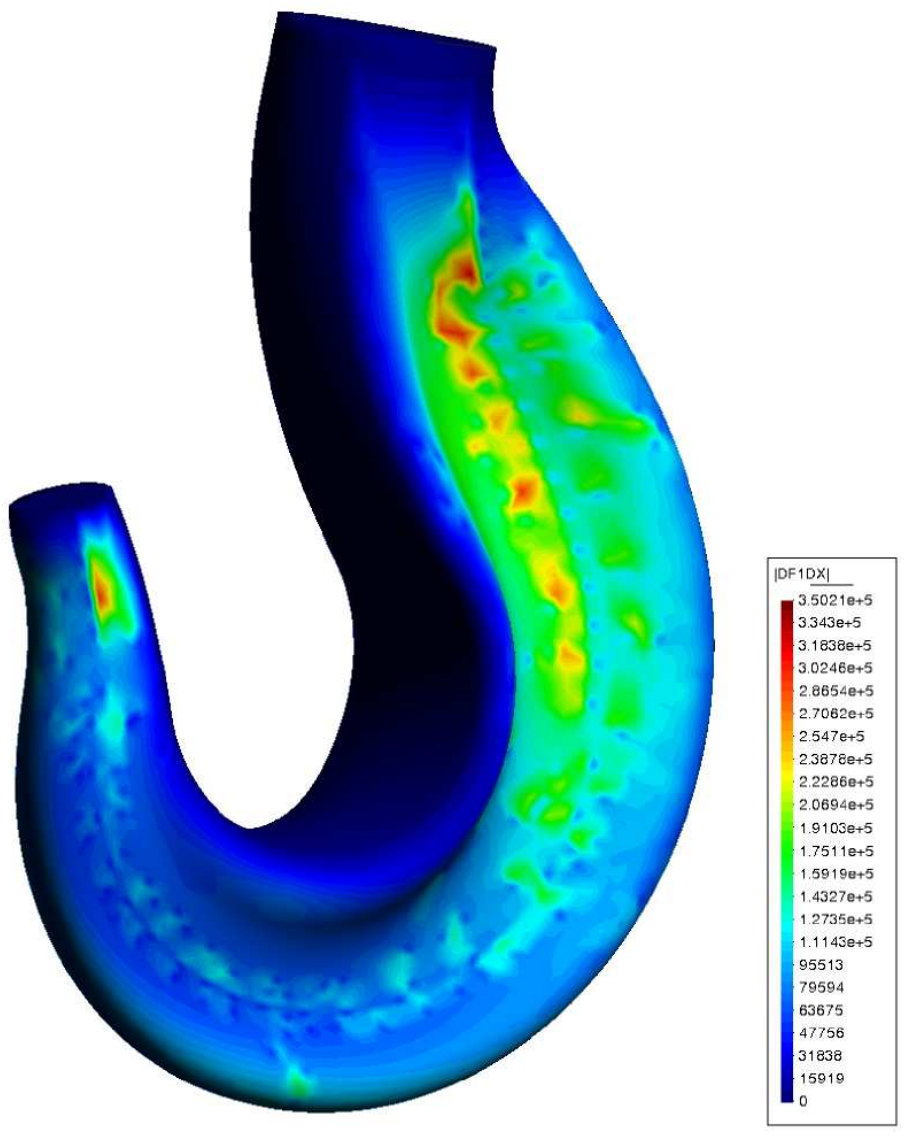

Figure 6: Sensitivity map at the tenth optimization iteration. 
The sensitivity map corresponding to the objective function in the tenth optimization iteration is shown in Fig. 6. Even without performing optimization, such a plot can be used to identify hot spots that have big influence on the objective which are indicated by red in Fig. 6.

\section{A API definition and usage}

This appendix provides a brief documentation and explanation of the API for the deterministic optimization and how those can be used.

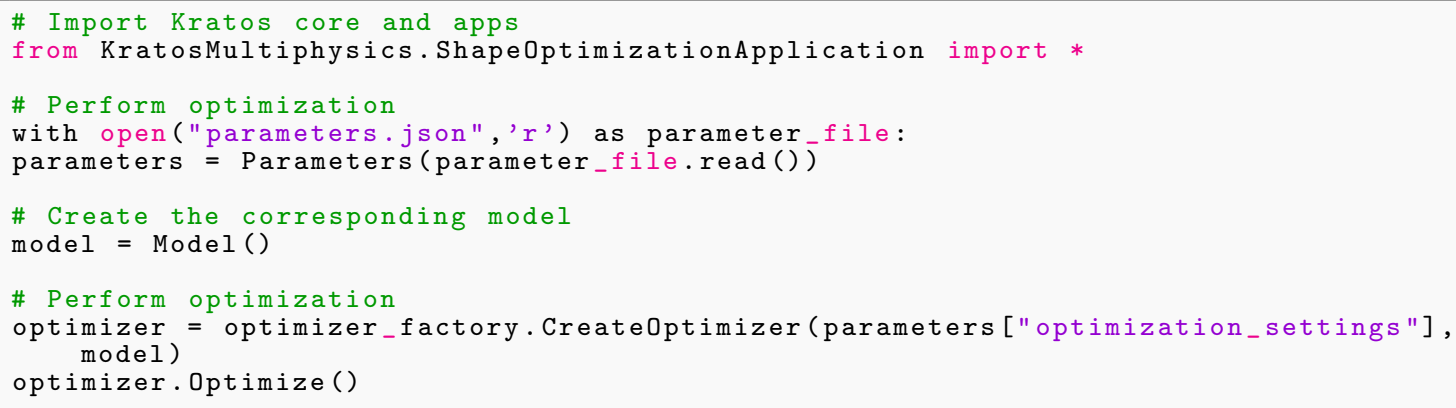

Figure 7: Example of the corresponding calls in the python level for the deterministic optimization algorithms using in KRATOS and an internal analyser. (taken from file ./src/run_test.py)

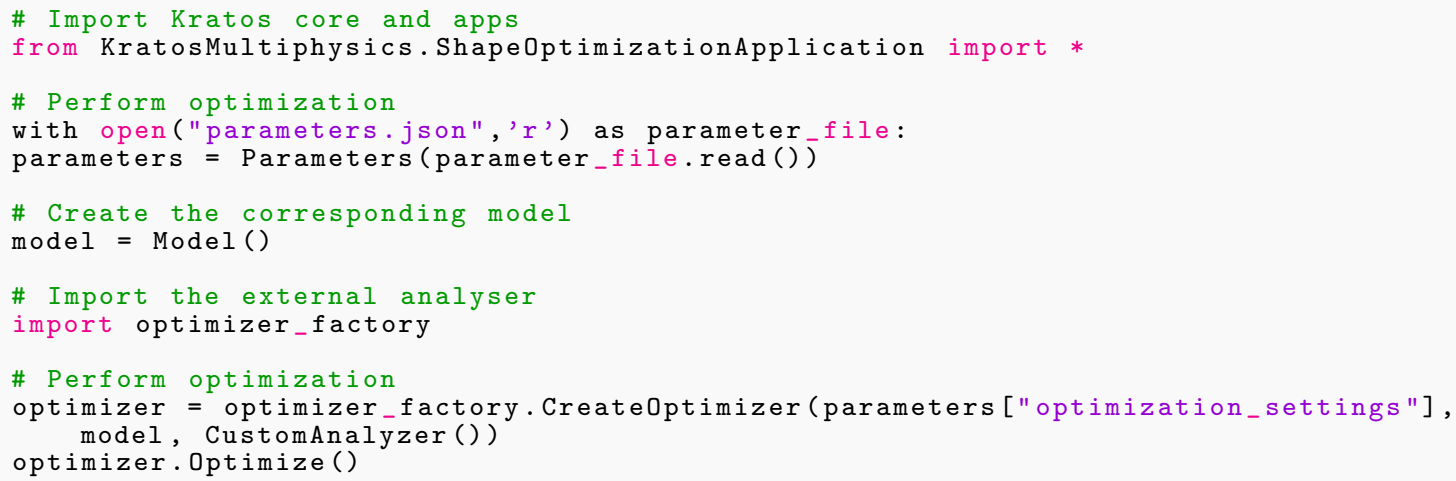

Figure 8: Example of the corresponding calls in the python level for the deterministic optimization algorithms using in KRATOS using an external analyser. (taken and slightly modified from file./src/run_test.py)

As standard in KRATOS Multiphysics software $\AA$, the simulation steps are controlled using a python layer. For the corresponding deterministic optimization algorithms, internal (implemented in KRATOS core) and/or external (third party) analysers can be used. This is shown in Figs. 7 and 8, respectively, where the external analyser is identified by CustomAnalyzer(). Concerning the internal analyser, the analysis stage within the corresponding class is shown in Fig. 9. 


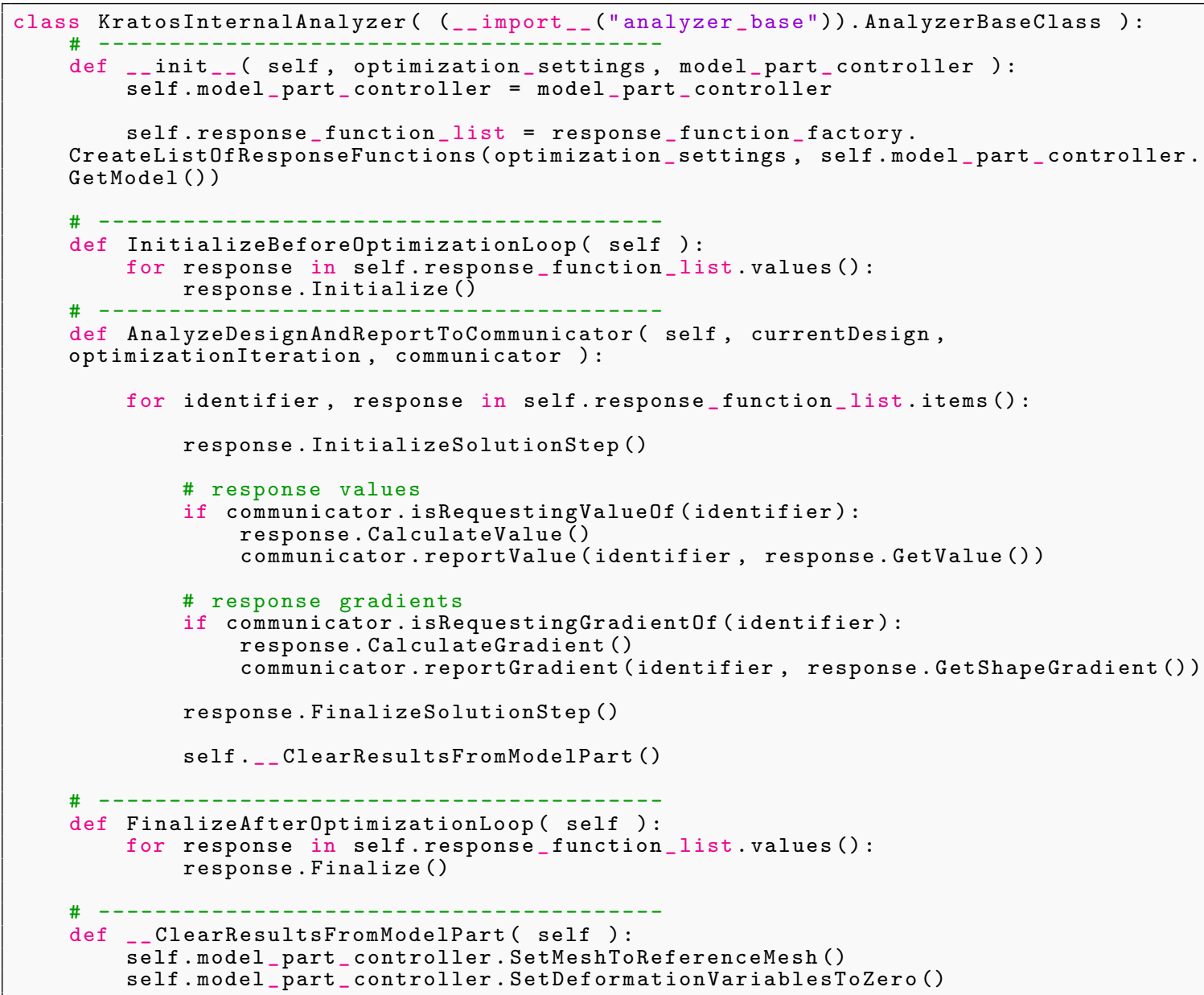

Figure 9: Class associated with the internal analyser in KRATOS. (taken from file ./src/analyzer_factory.py) 


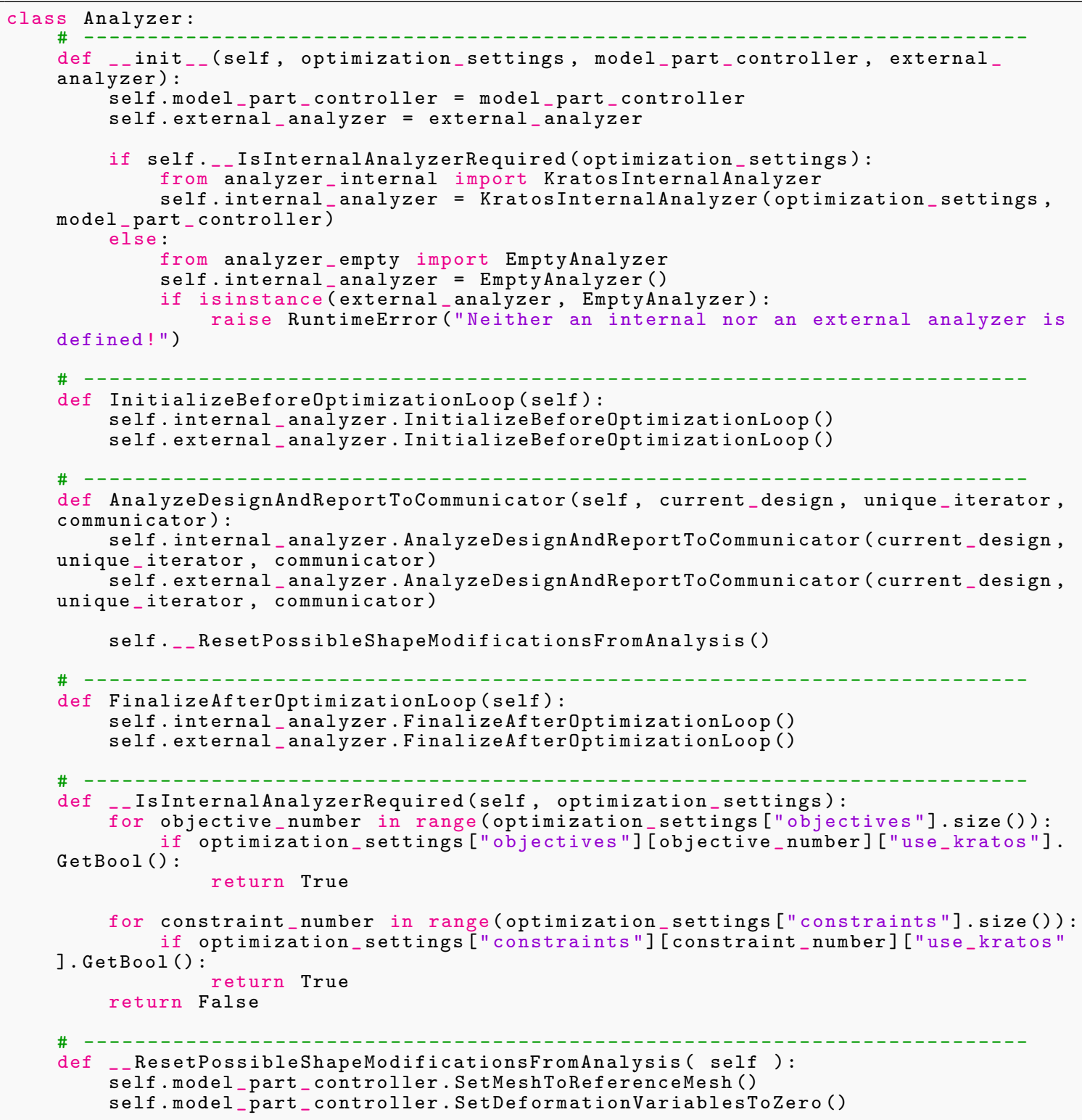

Figure 10: Class associated with the external analyser in KRATOS. (taken from file ./src/analyzer_factory.py) 
If one wishes to use a third party analyser, herein called external analyser, the class KratosInternalAnalyzer is employed, see Fig. 7.

The aforementioned class can then be then instantiated in the python layer as shown in Fig. 11, where functions such as __ObjectiveFunction, __ObjectiveGradient, etc. can be specialized.

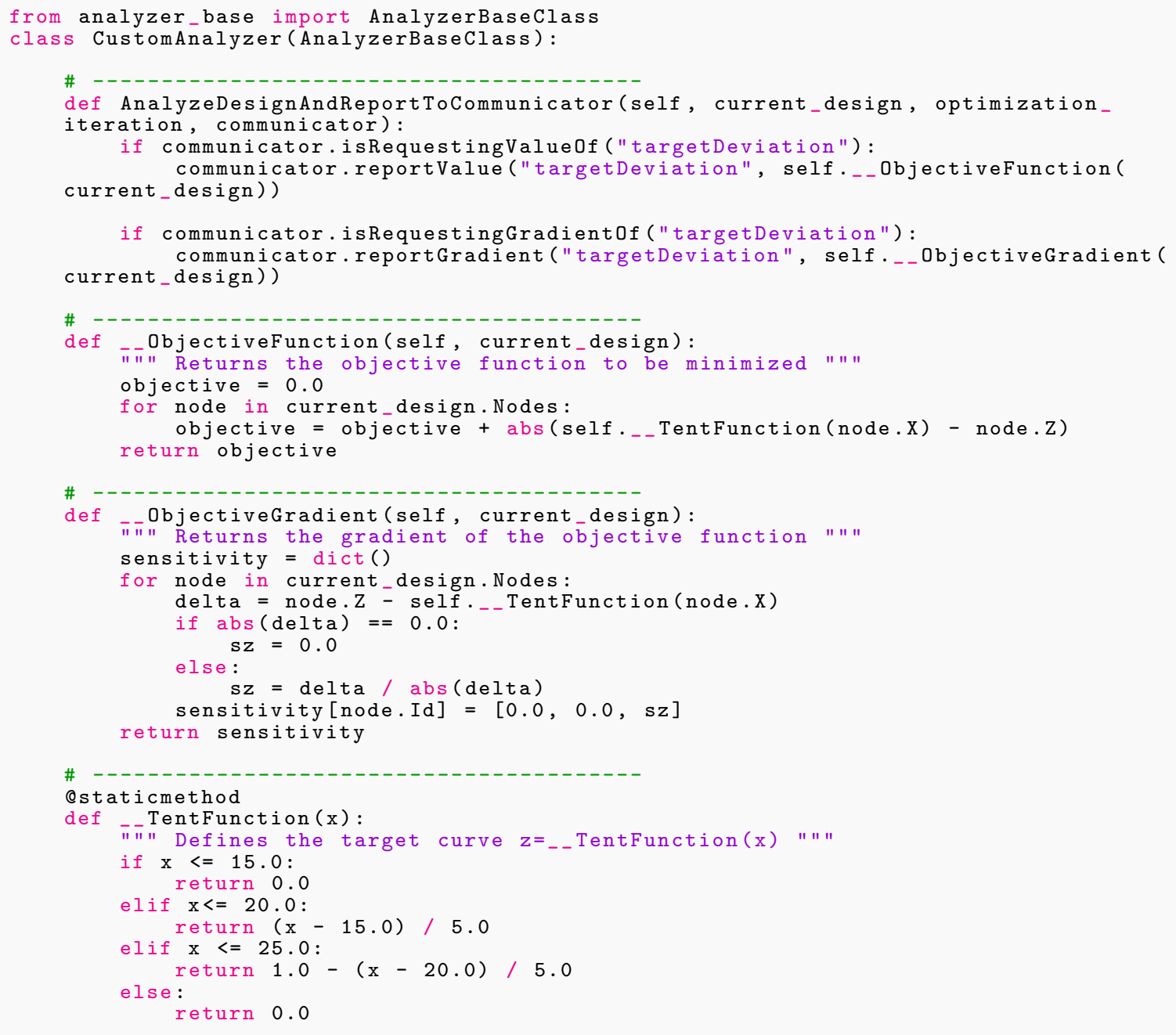

Figure 11: Specialization of the class associated with the external analyser in KRATOS. (taken from file./src/run_test.py)

Moreover, if one wishes to use an external analyser, entry ["optimization_settings"] in the parameters.json file need to be specified, see Fig. 12. Note that in case of employing an external analyser, the flag "use_kratos" needs to be set to false.

\section{References}

[1] D. Baumgärtner, A. Viti, A. Dumont, G. Carrier, and K.-U. Bletzinger. Comparison and combination of experience-based parameterization with vertex morphing in aerodynamic shape optimization of a forward-swept wing aircraft. In 17 th AIAA/ISSMO Multidisciplinary Analysis and Optimization Conference, American Institute of Aeronautics and Astronautics, 2016. 


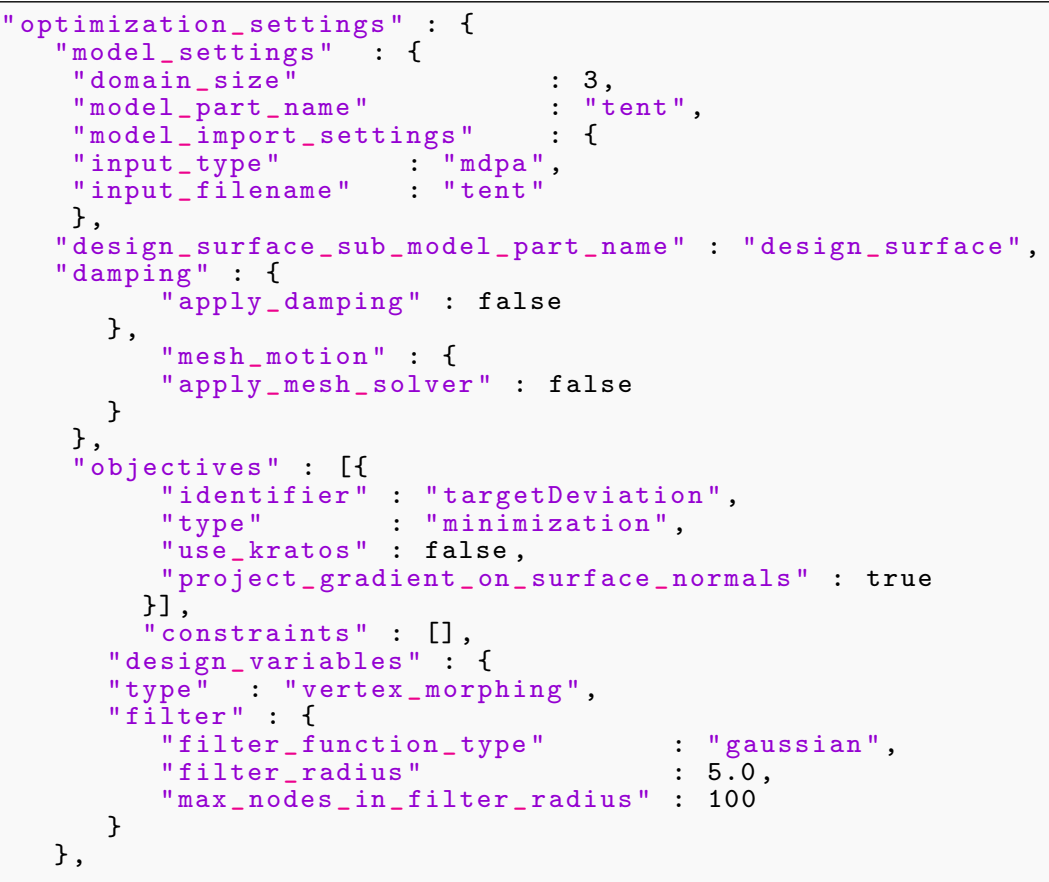

Figure 12: Specification of the optimization properties in parameters.json file when using an external analyser. (taken from file ./src/parameters.json)

[2] K.-U. Bletzinger. A consistent frame for sensitivity filtering and the vertex assigned morphing of optimal shape. Structural and Multidisciplinary Optimization, 49(6): 873-895, 2014.

[3] K.-U. Bletzinger. Shape optimization. Encyclopedia of Computational Mechanics Second Edition, R. B. and T. J. H. E. Stein, pages 1-42, 2017.

[4] M. Firl. Optimal shape design of shell structures. PhD thesis, Technische Universität München, 2010.

[5] R. T. Haftka and Z. Gürdal. Elements of structural optimization, volume 11. Springer Science \& Business Media, 2012.

[6] M. Hojjat, E. Stavropoulou, and K.-U. Bletzinger. The vertex morphing method for node-based shape optimization. Computer Methods in Applied Mechanics and Engineering, 268:494-513, 2014.

[7] V. Komkov, K. K. Choi, and E. J. Haug. Design sensitivity analysis of structural systems, volume 177. Academic press, 1986.

[8] H. Masching. Parameter Free Optimization of Shape Adaptive Shell Structures. PhD thesis, Technische Universität München, 2016.

[9] T. Wick. Fluid-structure interactions using different mesh motion techniques. Computers \& Structures, 89(13-14):1456-1467, 2011. 